Article

\title{
Efficient Oil/Water Separation Membrane Derived from Super-Flexible and Superhydrophilic Core-Shell Organic/Inorganic Nanofibrous Architectures
}

\author{
Zhi Liu ${ }^{1} * *$, Detao Qin ${ }^{2}$, Jianghui Zhao ${ }^{1}$, Quan Feng ${ }^{1}$, Zhengtao Li $^{3}$, Hongwei Bai ${ }^{3, *}$ and \\ Darren Delai Sun ${ }^{3, *}$ \\ 1 School of Textile and Garment, Anhui Polytechnic University, Beijing Road, Wuhu 241000, China; \\ zhaojh@ahpu.edu.cn (J.Z.); fengquan@ahpu.edu.cn (Q.F.) \\ 2 Energy Research Institute @ NTU, Interdisciplinary Graduate School, Nanyang Technological University, \\ Singapore 639798, Singapore; dqin001@e.ntu.edu.sg \\ 3 School of Civil and Environmental Engineering, Nanyang Technological University, Singapore 639798, \\ Singapore; ZLI018@e.ntu.edu.sg \\ * Correspondence: liuzhi@ahpu.edu.cn (Z.L.); baihw0001@gmail.com (H.B.); ddsun@ntu.edu.sg (D.D.S.); \\ Tel.: +86-134-8553-2640 (Z.L.); +65-9451-9756 (H.B.); +65-6790-6273 (D.D.S.)
}

Received: 9 May 2019; Accepted: 27 May 2019; Published: 3 June 2019

\begin{abstract}
To address the worldwide oil and water separation issue, a novel approach was inspired by natural phenomena to synthesize superhydrophilic and underwater superoleophobic organic/inorganic nanofibrous membranes via a scale up fabrication approach. The synthesized membranes possess a delicate organic core of PVDF-HFP and an inorganic shell of a $\mathrm{CuO}$ nanosheet structure, which endows super-flexible properties owing to the merits of PVDF-HFP backbones, and superhydrophilic functions contributed by the extremely rough surface of a $\mathrm{CuO}$ nanosheet anchored on flexible PVDF-HFP. Such an organic core and inorganic shell architecture not only functionalizes membrane performance in terms of antifouling, high flux, and low energy consumption, but also extends the lifespan by enhancing its mechanical strength and alkaline resistance to broaden its applicability. The resultant membrane exhibits good oil/water separation efficiency higher than $99.7 \%$, as well as excellent anti-fouling properties for various oil/water mixtures. Considering the intrinsic structural innovation and its integrated advantages, this core-shell nanofibrous membrane is believed to be promising for oil/water separation, and this facile approach is also easy for scaled up manufacturing of functional organic/inorganic nanofibrous membranes with insightful benefits for industrial wastewater treatment, sensors, energy production, and many other related areas.
\end{abstract}

Keywords: core shell; flexible; oil water separation; superhydrophilicity; underwater superoleophobicity

\section{Introduction}

Membrane separation is a popularly used technology for wastewater treatment, water purification, seawater desalination, and even oil/water separation [1-5]. Since its first development in the 1960s, polymer-based membranes have dominated the market in various applications field from the pharmaceutical/medical industry, food processing, gas separation, water purification and treatment, electronic devices, etc., with a huge number of membranes emerging in the market [6-8].

However, conventional polymer-based membranes synthesized by phase inversion processes not only involve a complicated process with lots of chemical consumption and waste gas/water generation, but also have a rough and hydrophobic surface, which is easy to be fouled by oil or other matter [9]. Membrane fouling owing to the accumulation of pollutants in the pore or on the surface of the membrane has become a bottleneck, limiting future membrane applications by painting it 
with an image of shorter lifespan, poorer water quality, lower water productivity, and higher energy consumption, which is contradictory to a sustainable and green development future [10,11].

An ideal separation membrane is featured with special properties such as flexibility for ease of application, superhydrophilicity for low fouling tendency and high productivity, and good mechanical strength for a longer lifespan. New material development with interdisciplinary innovation creates a lot of opportunities to make a breakthrough by integrating the advantages of well-designed next generation separation membranes [6] and continuous spinning technology [12]. To the best of our knowledge, few reports were published about the integration of organic backbone structures and inorganic surface properties with outstanding attributes and performances to address the severe oil/water separation issue.

Nature is a good school [13] and inspires us a lot to create many wonderful materials with promising applications to address practical engineering problems. In the past decades, special wettability membranes have been designed in combination with surface roughness and chemistry [14]. Jiang et al. firstly reported a superhydrophobic PTFE coated mesh film for oil/water separation [15]. Since then, membranes with superhydrophobicity have been intensively studied and applied to separate oils from water [16-18]. However, as water is denser than oil, it tends to form a barrier between the oil and hydrophobic membranes, preventing oil permeation through the membrane, and limiting the performance of oil and water separation to great extent. In addition, hydrophobic membranes always have an affinity to the oil phase, causing a high tendency of membrane fouling and clogging [19].

On the contrary, hydrophilic membranes, such as cellulose acetate membrane (HTI company) or emerging ceramic membranes, exhibit a low fouling tendency, while cellulose has low compatibility in extreme wastewater, such as extremely acidic or extremely alkaline conditions, resulting in shorter lifespans [20]. A ceramic membrane based on $\gamma-\mathrm{Al}_{2} \mathrm{O}_{3}$ or $\mathrm{SiC}$ with a good hydrophilic surface proved to be a promising solution for oil/water separation; however, ceramic membranes have at least three limiting factors: (a) brittle mechanical strength; (b) high-pressure pulse backwashing consumes a lot of energy and results in easy break up after a certain operation cycle, and as such, many researchers and applications prefer material flexibility [21,22]; and (c) membrane pore size can be increased after a certain time operation under huge pressure scouring, which limits its rejection capability [23].

Very recently, inspired by the antiwetting properties of oil droplets on fish scales, a new idea of underwater superoleophobic surfaces was proposed [24]. Following this concept, many superhydrophilic in air and superoleophobic in water surfaces have been achieved in various ways $[25,26]$. Among them, two main approaches have been applied to construct antiwetting surfaces in water. One is surface fictionalization by hydrogel coating. This strategy is a typical and feasible approach for fabricating hydrophilic membranes. For example, Feng et al. [27] fabricated a hydrogel coated mesh with underwater superoleophobicity to separate the immiscible oil/water mixture. Liu et al. [28] coated inorganic oxides such as cooper oxide to construct a low adhesive superoleophobic surface. However, the coated membranes have obvious drawbacks of the weakened or unstable hydrophilic property under harsh conditions [17,29]. The other popular method is grafting nano-structures on metal mesh. For example, $\mathrm{Zhang}$ et al. [30] assembled $\mathrm{TiO}_{2}$ nanoparticles on the surface of stainless steel mesh separating water from an oil/water mixture effectively. For a further step, Zhang and co-workers also fabricated a nanowire-haired $\mathrm{Cu}(\mathrm{OH})_{2}$ on a copper mesh, and this resulting membrane was capable of separating oil/water mixtures with high efficiency under alkaline conditions [31]. However, these regenerated membranes on metal mesh have many disadvantages such as large pore size and poor flexibility, weakening the applications in oil/water separation [32].

Therefore, the development of a highly efficient, flexible membrane with long-term usability, low membrane fouling and feasible fabrication process is greatly desired. The core-shell structured microfiltration membrane with hydrophilic surface was reported in our previous study and the resulting membrane shows good microfiltration performance [33]. In the present study, we aim to (1) the detailed fabrication process of an organic/inorganic superflexible and superhydrophilic nanofiber 
membrane; (2) the properties (wettability, flexibility, mechanical property) of the resulting membrane; (3) the oil/water separation performance of the resulting membrane; and (4) tentative conclusions of formation mechanisms of the resulting membrane.

\section{Experimental Section}

\subsection{Materials}

Poly(vinylidene fluoride-co-hexa-fluoropropylene) (PVDF-HFP, $M \mathrm{w}=400,000$ ) and cupric acetate monohydrate were supplied by Aladdin Industrial Corporation, Shanghai, China. N,N-dimethyl formamide (DMF), ammonia water, and acetone were all purchased from Sinopharm Chemical Reagent Co., Ltd. (Suzhou, China). All reagents were used as obtained without further treatment.

\subsection{PVDF-HFP/Cu(CH $\left.\mathrm{CH}_{3} \mathrm{COO}\right)_{2}$ Nanofibrous Membrane Prepared by Electrospinning Process}

PVDF-HFP (11 wt \%) was dissolved in binary solvent of DMF/acetone (weight ratio of 5:5) at room temperature for $4 \mathrm{~h}$. Then, the cupric acetate was added into the as-prepared solutions with different weight ratios of cupric acetate monohydrate/PVDF-HFP (1:2, 1:3, 1:6). Then the mixed solutions were stirred for $20 \mathrm{~min}$ under $70^{\circ} \mathrm{C}$ to achieve blue solutions. During the electrospinning process, the collector distance was kept at $25 \mathrm{~cm}$ and a voltage of $25 \mathrm{kV}$ was applied. The ambient relative humidity $(\mathrm{RH})$ and temperature used in the spinning process were $50 \pm 2 \%$ and $25 \pm 2{ }^{\circ} \mathrm{C}$, respectively. Lastly, the PVDF-HFP/Cu( $\left(\mathrm{CH}_{3} \mathrm{COO}\right)_{2}$ nanofibrous membrane was fabricated and named as $\mathrm{P} / \mathrm{Cu}\left(\mathrm{CH}_{3} \mathrm{COO}\right)_{2}$ membrane.

\subsection{PVDF-HFP/CuO Nanofibrous Membrane Prepared by Heating Process}

The as-prepared $\mathrm{P} / \mathrm{Cu}\left(\mathrm{CH}_{3} \mathrm{COO}\right)_{2}$ membrane was put into an electronic oven under $120^{\circ} \mathrm{C}$ for $24 \mathrm{~h}$. The heating process was carried out to convert $\mathrm{Cu}\left(\mathrm{CH}_{3} \mathrm{COO}\right)_{2}$ into $\mathrm{CuO}$, acting as the seed for $\mathrm{CuO}$ nanosheet nucleation and crystallization in the following steps. After reaction, the PVDF-HFP/CuO nanofibrous membranes were fabricated and named as $\mathrm{P} / \mathrm{CuO}$ membrane.

\subsection{PVDF-HFP/CuO-Nanosheet Nanofibrous Membrane Prepared by Hydrothermal Process}

The as-synthesized membranes after the heating process were immersed in the prepared growth media (Figure S5). The heat-resistant glass bottles were used as hydrothermal reactors to hold the growth media and the $\mathrm{P} / \mathrm{CuO}$ membrane. Hydrothermal reactions occurred in an electronic oven under different temperature $\left(40,60\right.$, and $80^{\circ} \mathrm{C}$, respectively). The resulting nanofibrous membranes were cleaned by deionized water and then dried in oven at $40^{\circ} \mathrm{C}$ for $5 \mathrm{~h}$ to remove the residual solvent. Thereafter, the membranes equilibrated at room conditions in air for several days before the water contact angle (CA) measurements. After the hydrothermal process, the PVDF-HFP/CuO-nanosheet nanofibrous membrane was produced and named as $\mathrm{P} / \mathrm{CuO}$-nanosheet membrane.

\subsection{Characterizations}

The resulting membrane morphology was observed by an FE-SEM (JEOL 7600F, Akishima, Japan) at $20^{\circ} \mathrm{C}, 65 \mathrm{RH}$. The fiber diameters were calculated by measuring at least 100 fibers at random using the Image J program. Fourier transform infrared spectroscopy (FTIR, Nicolet 5700, Waltham, MA, USA) and X-ray diffraction (XRD, X' Pert-pro MRD, Philips, Almelo, The Netherlands) were used to study the structure of the resulting nanofibrous membranes. In the testing process, the XRD scan rate was set as $4^{\circ} / \mathrm{min}$ with the $2 \theta$ ranging from 10 to $80^{\circ}$, and the FTIR spectrum ranged from 400 to $4000 \mathrm{~cm}^{-1}$. The FTIR and XRD results were corrected by the Savitzky-Golay method with the points of window of 20 and the polynomial order of 1.

The wetting abilities of the resulting membranes were characterized by an optical CA meter system (Krüss DSA100, Krüss, Hamburg, Germany). For underwater oil CA measurement, the membrane was firstly immersed in water, then a trichloromethane droplet $(6 \mu \mathrm{L})$ was dropped onto the membrane. 
The final values were the average of five droplets based on different locations. An Olympus TH4-200 microscope was used to capture the optical microscopy images before and after separation.

A thermogravimetric analyzer (TGA (Symonston, Australia), TGA-4000, PerkinElmer, Waltham, MA, USA) was used to examine the water content in the filtrated product. In this process, the sample was heated from room temperature to $110^{\circ} \mathrm{C}$ at a rate of $5^{\circ} \mathrm{C} / \mathrm{min}$ and held for $60 \mathrm{~min}$. The separation efficiency was calculated by the following equation:

$$
R=\left(1-W_{2} / W_{1}\right) \times 100 \%
$$

where, the $R$ is the rejection rate, $W_{2}$ is the oil percentage after separation, and $W_{1}$ is the oil percentage before separation.

The size distribution of oil droplets in the mixture was measured by a laser particle analyzer (Mastersizer-2000, Malvern, UK). The oil-water mixtures were prepared by mixing $n$-hexane, olive oil, cooking oil, and lubricant oil with water in a weight ratio of 1:10, respectively. The nitrogen provided the pressure ( 0.3 bar in the present study). During the separation experiment, the mixtures were poured into the filtration cup and separated by the membrane at the cup bottom. Then the flux was obtained by the following equation:

$$
J=\frac{V}{t \times s}
$$

where $J$ is the flux $\left(\mathrm{Lm}^{-2} \mathrm{~h}^{-1}\right), V$ is the mixture volume, $t$ is the time, and $s$ is the resulting membrane area in the separation experiment.

\section{Results and Discussion}

\subsection{Evolution of P/CuO-Nanosheet Membrane Formation Process}

In this study, we introduced a facile process including an in-situ growth method to construct a delicate core-shell structured nanofibrous membrane. To vividly explain this process, a schematic diagram was drawn to explain the membrane formation evolution, as seen in Figure 1. Clearly, this integrated facile approach covers three major steps with different functions. Step 1 is continuous spinning of the PVDF-HFP nanofiber membrane by needle-disk electrospinning. Nanofiber high throughput is crucial to practical application [34]. Therefore, needle-disk electrospinning, which has demonstrated fabricating nanofiber with high quality and high throughput under a lower applied voltage, was applied to fabricate the nanofibrous membrane (Figure S1, Supplementary Information (SI)) [35,36]. Here, the modified electrospinning method was applied to aim at high throughput of the as-prepared membranes with greater uniformity compared to the conventional single-needle spinning process. After this procedure, $\mathrm{P} / \mathrm{Cu}\left(\mathrm{CH}_{3} \mathrm{COO}\right)_{2}$ membrane was fabricated (Figure 1). In Step 2, the heating process is helpful to form $\mathrm{CuO}$ nanoseeds on PVDF-HFP nanofibers, which is the crucial step for the formation of $\mathrm{CuO}$ nanoseeds to facilitate the nucleation and crystallization of $\mathrm{CuO}$ nanosheets [37]. The as-prepared membrane was heated in an oven at a temperature, inducing a cupric acetate reaction. After the reaction, the cupric acetate turned to cooper oxide and spread on the surface of the PVDF-HFP nanofiber (Figure 1). In Step 3, a low temperature hydrothermal reaction is intended to graft/anchor $\mathrm{CuO}$ nanosheets on PVDF fibers for stable applications. Previous reports of hydrothermal temperature were higher than $100{ }^{\circ} \mathrm{C}$, even up to hundreds of degrees, which is not economical for scale up production owing to high energy consumption $[38,39]$. By contrast, the hydrothermal temperature in this study was as low as $60^{\circ} \mathrm{C}$, which was an energy saving approach and easy for scale up production. In this hydrothermal process, $\mathrm{CuO}$ nanosheets first nucleate on the well-formed nanoseeds and then crystallize into nanosheets with specific crystal properties. Rather than a physical mixing process, the chemical reaction initiates the nucleation, and crystal formation of $\mathrm{CuO}$ nanosheets enhances the anchoring affinity between $\mathrm{CuO}$ and PVDF-HFP nanofibers, which leads to excellent mechanical strength properties of the resultant membrane (Figure 1). 


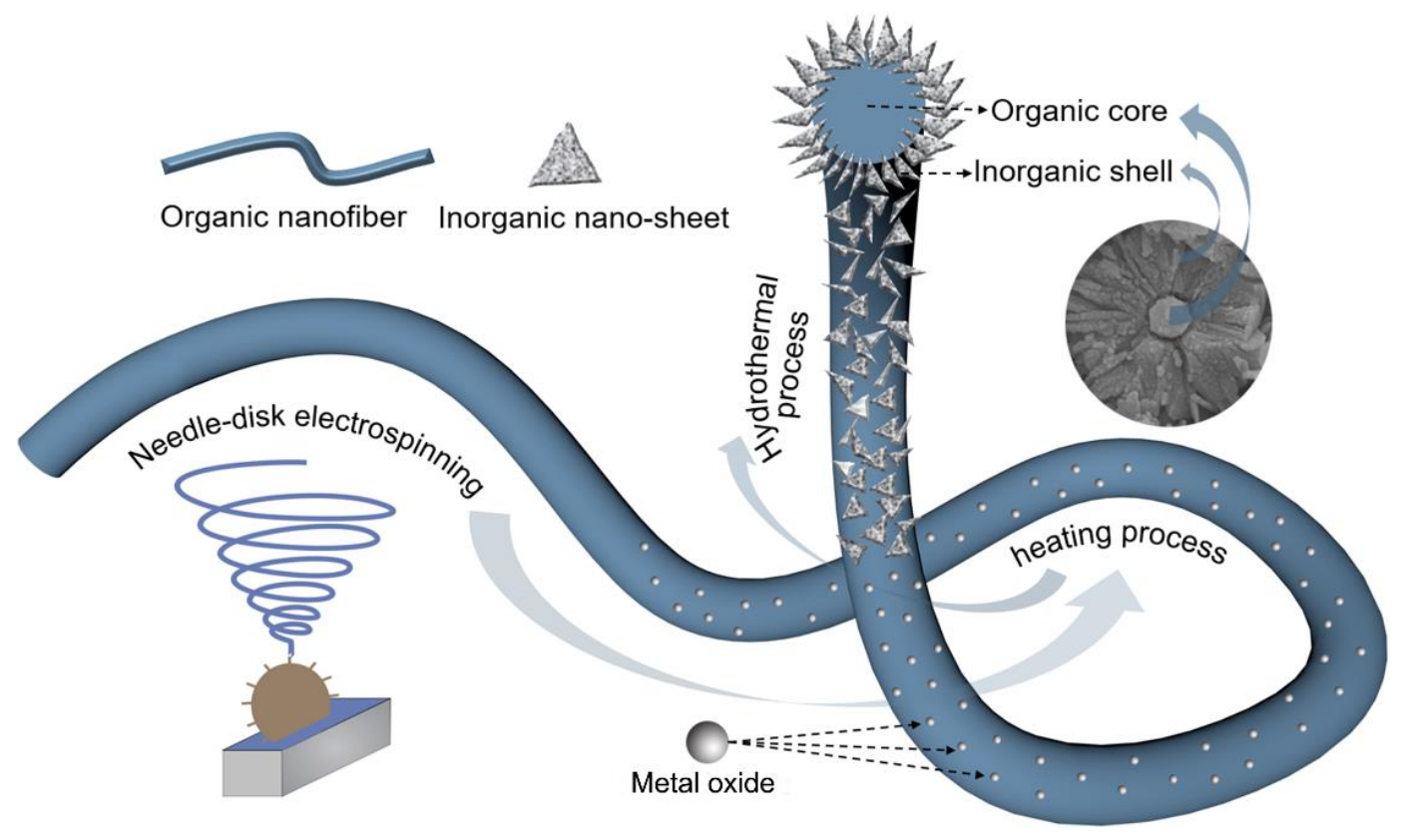

Figure 1. Schematic diagram of the evolution of the $\mathrm{P} / \mathrm{CuO}$-nanosheet membrane.

\subsection{The Morphology and Structure of the Resulting Membranes}

The morphology of the resultant membranes after each step are present in Figure 2. Obviously, the hydrophilicity property was enhanced gradually after heating (burning off organic residuals and forming $\mathrm{CuO}$ nanoseeds) and hydrothermal process (forming $\mathrm{CuO}$ nanosheet crystals). As shown in Figure 2a, the color of as-spun membrane was light blue, which was attributed to the cupric acetate. The resulting PVDF-HFP/cupric acetate nanofibers showed a smooth surface (Figure 2b) with fiber diameter of $198 \pm 24 \mathrm{~nm}$ and narrow diameter distribution from $140 \mathrm{~nm}$ to $280 \mathrm{~nm}$ (Figure S2). Due to the PVDF-HFP hydrophobicity, the resulting composite membrane showed good hydrophobic properties with water CA $145.8^{\circ}$. After the heating process, the color changed to brown (Figure $2 \mathrm{~d}$ ), and the fiber diameter decreased to $161 \pm 21 \mathrm{~nm}$ (Figure S2) owing to fiber shrinking and remodeling after residual solvent evaporation under heating. Meanwhile, the water CA decreased to $138.3^{\circ}$ (Figure 2f). Obviously, the changed color and decreased water $\mathrm{CA}$ indicated the formation of $\mathrm{CuO}$ on the PVDF-HFP surface after the heat reaction. Finally, the color changed to black, and a few nanometers in thickness and 100-300 $\mathrm{nm}$ in length nanosheet structure was achieved (Figures $2 \mathrm{~h}$ and $3 \mathrm{a}$ ), suggesting the growth of the $\mathrm{CuO}$ nanosheet after hydrothermal process. Moreover, the wettability of the resulting membrane changed dramatically from $138.3^{\circ}$ to $0^{\circ}$ (Figure $2 \mathrm{i}$ ), which was as a result of the formation of a $\mathrm{CuO}$ nanosheet on the PVDF-HFP membrane. In order to reveal the effect of hydrothermal temperature on the resultant membrane, a systematic study with various $\mathrm{P} / \mathrm{CuO}$ membranes was synthesized under different temperatures, with results showing in Figure S3, so as to determine the optimal hydrothermal reaction temperature. The experimental results demonstrated that the weight ratio of PVDF-HFP/cupric acetate and hydrothermal temperature had a great impact on the membrane morphology. With the decrease of the cupric acetate ratio, nanosheets decreased on the nanofiber surface, and no apparent nanosheets were observed when the weight ratio of cupric acetate/PVDF-HFP was 1:6 (Figure S3). A relatively high temperature of $40^{\circ} \mathrm{C}$ did not lead to obvious membrane morphology (Figure S4). However, further temperature increases up to $80^{\circ} \mathrm{C}$ resulted in tight nanosheets growing on the fiber surface because of the increased growth rate of nanosheets under $80^{\circ} \mathrm{C}$ (Figure S4). Therefore, a growth temperature of $60^{\circ} \mathrm{C}$ presented a mild growing process (Figure S4). 


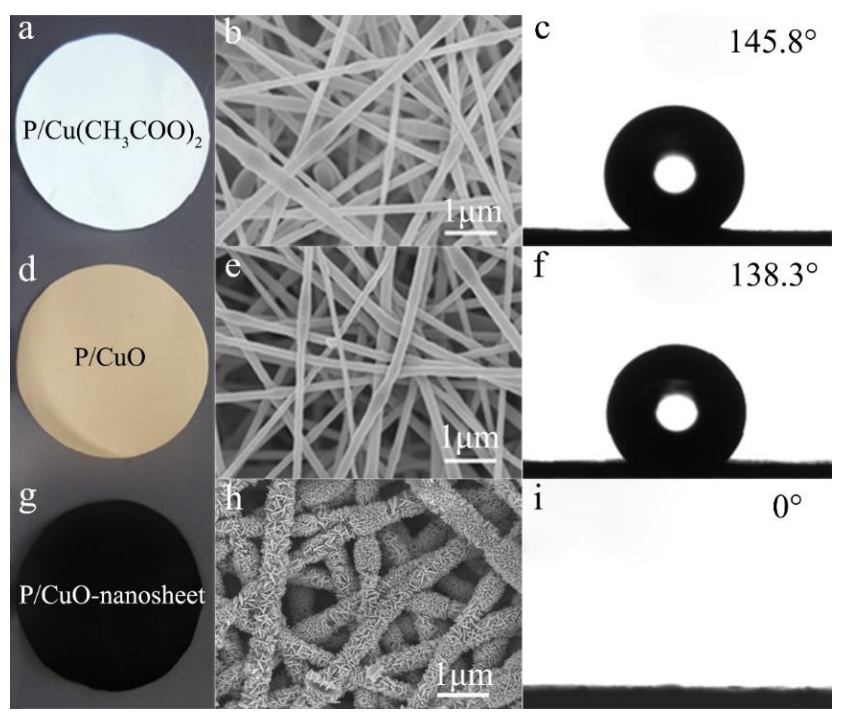

Figure 2. The optical image of (a) $\mathrm{P} / \mathrm{Cu}\left(\mathrm{CH}_{3} \mathrm{COO}\right)_{2}$, (d) $\mathrm{P} / \mathrm{CuO}$, and (g) $\mathrm{P} / \mathrm{CuO}$-nanosheet membranes; the morphology of (b) $\mathrm{P} / \mathrm{Cu}\left(\mathrm{CH}_{3} \mathrm{COO}\right)_{2}$, (e) $\mathrm{P} / \mathrm{CuO}$, and (h) $\mathrm{P} / \mathrm{CuO}$-nanosheet membranes; the wettability of (c) $\mathrm{P} / \mathrm{Cu}\left(\mathrm{CH}_{3} \mathrm{COO}\right)_{2}$, (f) $\mathrm{P} / \mathrm{CuO}$, and (i) $\mathrm{P} / \mathrm{CuO}$-nanosheet membranes.

The cross-section morphology demonstrates the core-shell structure. As shown in Figure 3d, the membrane thickness was about $90 \mu \mathrm{m}$. It can be seen from Figure $3 e$ that nanosheets covered all the surface of PVDF-HFP nanofibers, constructing a PVDF-HFP core and nanosheet shell. The amplified Figure $3 \mathrm{f}$ further demonstrates the core-shell structure. The brittle fracture interface under nitrogen conditions shows the inner PVDF-HFP nanofiber and external nanosheet (Figure 3f). This delicate structure integrates the advantages of the organic polymer with the inorganic nanosheet. The FTIR spectrum indicated that the-COO-radical existing in the $\mathrm{P} / \mathrm{Cu}\left(\mathrm{CH}_{3} \mathrm{COO}\right)_{2}$ membrane reacted after the heating process, as seen by the disappearance of $1572 \mathrm{~cm}^{-1}$ after the heating process (Figure 3b) [40]. Taking all the above factors like color, wettability change, and the FTIR spectrum change after the heating process into account, it was concluded that cupric acetate reacted and transformed into copper oxide. We speculated that cupric acetate reacted with water in the oven and changed to $\mathrm{CuO}$ and $\mathrm{CH}_{3} \mathrm{COOH}$. Then, the $\mathrm{CH}_{3} \mathrm{COOH}$ volatilized at $120{ }^{\circ} \mathrm{C}$, leaving $\mathrm{CuO}$ nanoseeds spreading on the surface of PVDF-HFP nanofibers (Figure 3).

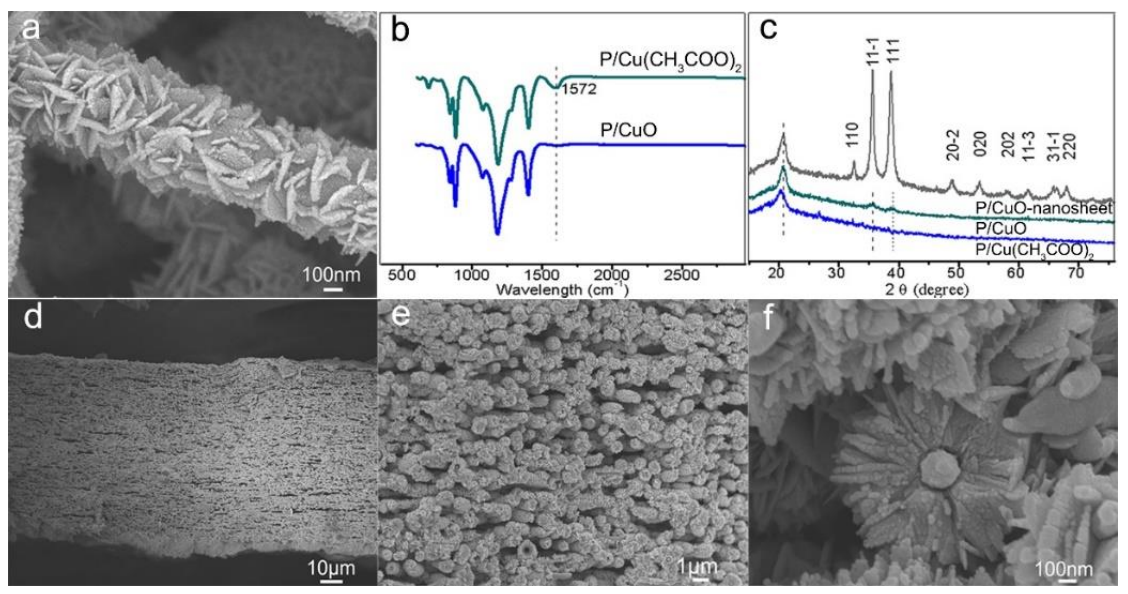

Figure 3. Morphology and structure of core-shell $\mathrm{P} / \mathrm{CuO}$-nanosheet membrane: (a) the morphology amplified by FE-SEM; (b) the FTIR spectrum of $\mathrm{P} / \mathrm{Cu}\left(\mathrm{CH}_{3} \mathrm{COO}\right)_{2}$ and $\mathrm{P} / \mathrm{CuO}$ membrane; (c) the XRD spectrum of $\mathrm{P} / \mathrm{Cu}\left(\mathrm{CH}_{3} \mathrm{COO}\right)_{2}, \mathrm{P} / \mathrm{CuO}$, and $\mathrm{P} / \mathrm{CuO}$-nanosheet membranes; $(\mathbf{d}-\mathbf{f})$, the cross-section morphology with different magnification $(\mathbf{d}) \times 700$, (e) $\times 5000$, and $(\mathbf{f}) \times 60,000$. 


$$
\mathrm{Cu}\left(\mathrm{CH}_{3} \mathrm{COO}\right)_{2}+\mathrm{H}_{2} \mathrm{O} \rightarrow \mathrm{CuO}+2 \mathrm{CH}_{3} \mathrm{COOH} \uparrow
$$

The XRD pattern further confirmed the growth of $\mathrm{CuO}$ on PVDF-HFP nanofiber surface. As shown in Figure $3 c$, after the heating process, the two peaks at $35.7^{\circ}$ and $38.8^{\circ}$ corresponded to the characteristic peak of $\mathrm{CuO}$ [41]. What is more, after the hydrothermal process, the peaks at $32.6^{\circ}, 35.7^{\circ}, 38.8^{\circ}, 48.9^{\circ}$, $53.5^{\circ}, 58.1^{\circ}, 61.7^{\circ}, 66.1^{\circ}$, and $68.1^{\circ}$ indicated the monoclinic system $\mathrm{CuO}$ crystals, which agreed with the values in the standard card (JCPDS) [42,43]. Moreover, the extremely strong peaks suggested high crystallinity of the $\mathrm{CuO}$ nanosheet on the PVDF-HFP backbone (Figure 3c).

\subsection{The Wettability and Mechanical Properties of the P/CuO Nano-Sheet Membrane}

The extremely rough $\mathrm{CuO}$ nanosheet on the fiber surface endows the membrane special wettability. As shown in Figure 4a, when a water droplet contacted the membrane surface, the droplet spread quickly and a zero water CA was achieved. Interestingly, the entire process was completed within $256 \mathrm{ms,}$ indicating superior water wetting properties of the membrane (Figure 4a). The outstanding wettability in air always leads to a good underwater oil anti-wetting property [32], which, as confirmed by Figure $4 \mathrm{~b}$, the underwater oil CA reached $152.4^{\circ}$. The superhydrophilic and underwater superoleophobic properties were attributed to the extra rough structure resulting from $\mathrm{CuO}$ nanosheets on the membrane and the hydrophilic nature of $\mathrm{CuO}$. When the nanosheet membrane was immersed in water, water was immediately trapped into the $\mathrm{CuO}$ nanosheet structures, forming an oil/water/solid interface in the presence of oil (Figure 4c). In other words, the trapped water acted as a repulsive layer for oils to make contact with the membrane directly. Owing to such surface properties, the resulting membrane allowed only the water to pass through and suggested a potential application in oil/water separation.

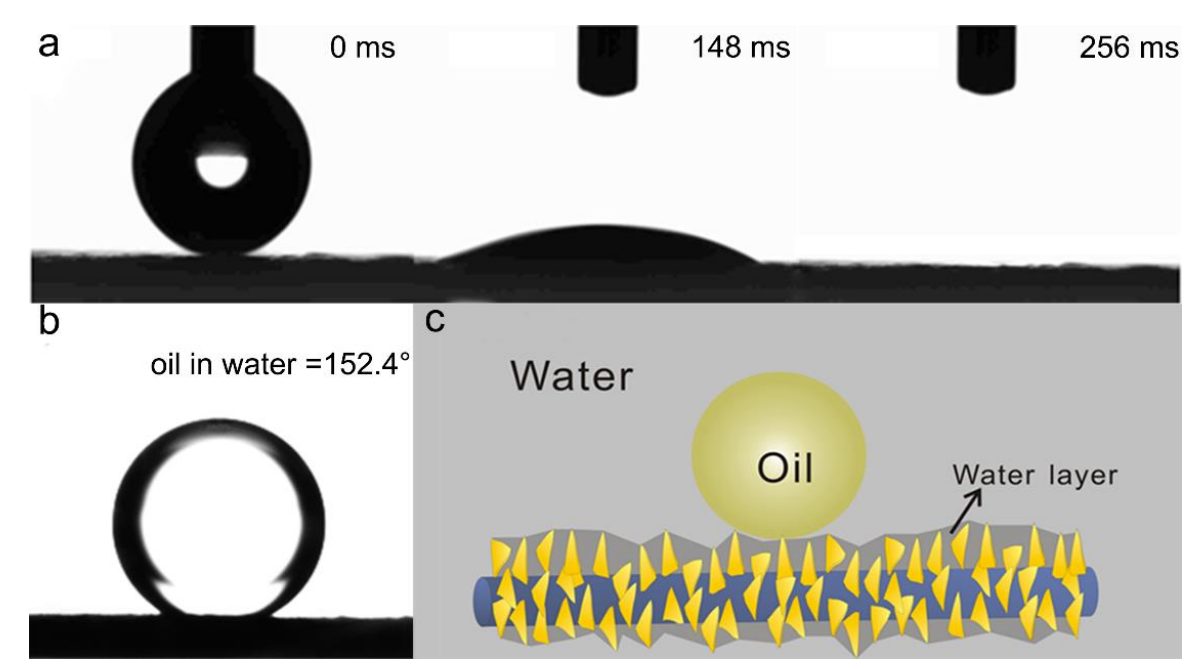

Figure 4. The wettability of the resulting P/CuO-nanosheet membrane: (a) the dynamic process of water droplets spreading on the membrane; (b) the underwater oil CA of the membrane; (c) the schematic diagram of the proposed water layer between the oil/water/solid interfaces.

Previous metal-based membranes always have poor flexible though they had good underwater oleophobicity. The membranes, in our study, possessed a polymer core and an inorganic shell, making it excellent in terms of flexibility as well as good underwater oleophobicity. As presented in Figure $5 \mathrm{a}, \mathrm{b}, \mathrm{d}, \mathrm{e}$, the resulting membrane could be folded for various desired shapes. The supporting video further confirmed the high flexibility of the membrane by blowing the membrane with a hair dryer. The $\mathrm{P} / \mathrm{Cu}\left(\mathrm{CH}_{3} \mathrm{COO}\right)_{2}$ membrane showed good mechanical properties with the stress at break 6.77 MPa and elongation at break $135.79 \%$. However, the heating process weakened the mechanical properties, resulting in the stress at break $2.49 \mathrm{MPa}$ and elongation at break $115.68 \%$ for the $\mathrm{P} / \mathrm{CuO}$ membrane. Interesting, the stress at break of $\mathrm{P} / \mathrm{CuO}$-nanosheet membrane increased to $6.44 \mathrm{MPa}$ 
and elongation at break decreased to $66.23 \%$. It can be speculated that the formed CuO-nanosheet enhanced the linkage force between the fibers, favoring the increase of stress at break and the decrease of elongation at break.
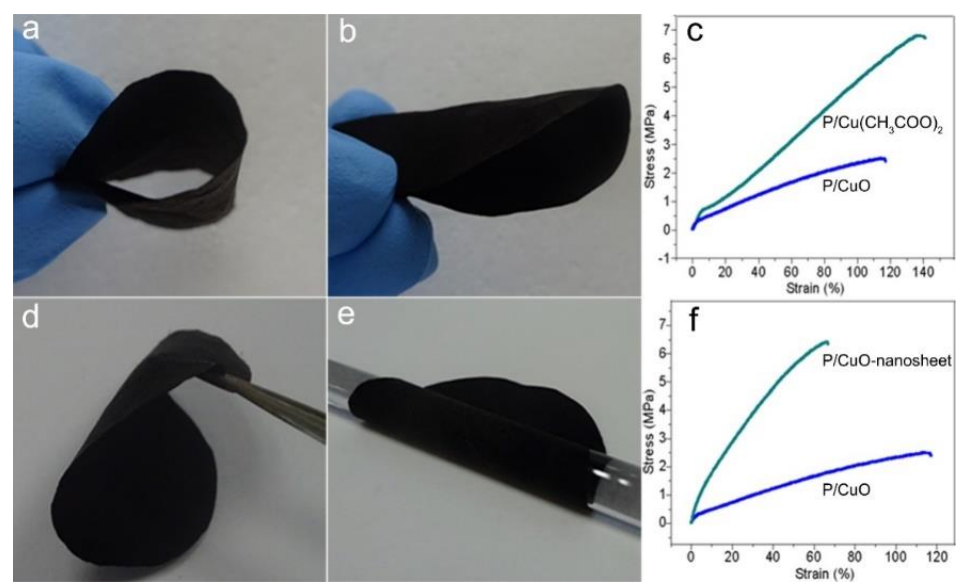

Figure 5. The optical image of $\mathrm{P} / \mathrm{CuO}$-nanosheet membrane with alterable shape $(\mathbf{a}, \mathbf{b}, \mathbf{d}, \mathbf{e})$; $(\mathbf{c}, \mathbf{f})$, the mechanical properties of $\mathrm{P} / \mathrm{Cu}\left(\mathrm{CH}_{3} \mathrm{COO}\right)_{2}, \mathrm{P} / \mathrm{CuO}$ membranes, and $\mathrm{P} / \mathrm{CuO}$-nanosheet membranes.

In addition, the membrane held good stress at break, sacrificing with the strain at break after the whole process (Figure 5c,f). Therefore, the super flexibility accompanied by an actable mechanical property broadens the membrane for practical industrial applications.

\subsection{The Membrane Performance for Oil/Water Separation}

For the oil/water separation process, the oil/water mixtures were separated by a home-made setup (Figure S6) with the results being illustrated in Figure 6. As shown in Figure 6a, the model oil/water mixture (olive oil) showed the size distribution of oil droplets from 1 to $40 \mu \mathrm{m}$, while previous metal-based membranes could not be separated with a high rejection rate due to the large membrane pore size [44]. The size distribution of oil droplets was further characterized by the microscopy images, which were in accordance with the particle analyzer measurement results (Figure 6c, left). After separation, a clear filtrate was collected (Figure 6c, right) with a high separation efficiency higher than $99.8 \%$ (Figure $6 \mathrm{~b}$ ), highlighting that the membrane was effective in separating oil/water mixtures with oil droplets sizes large than $1 \mu \mathrm{m}$.
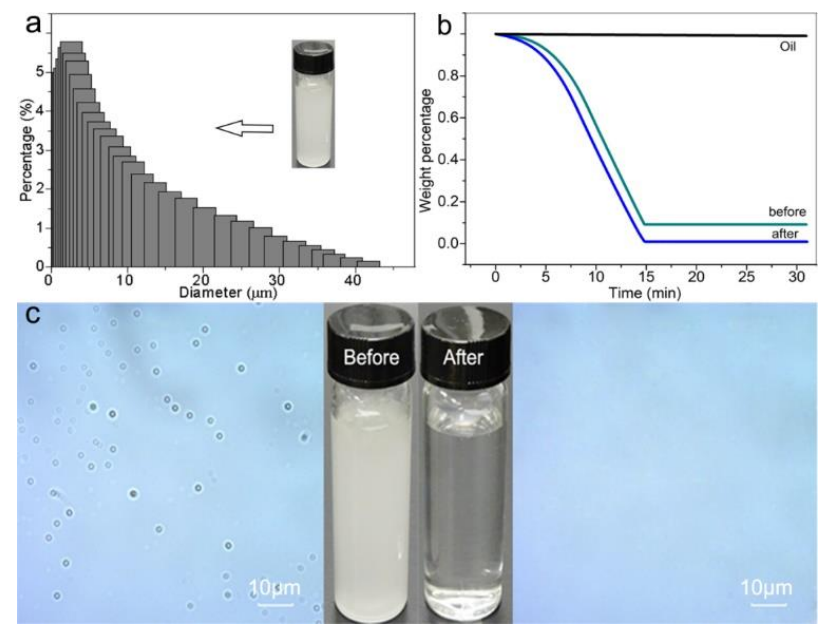

Figure 6. (a) the size distribution of oil droplets in mixture (olive oil); (b) the TGA curve of olive oil (as comparison), before and after separation; (c) the macroscope image of the solution before and after separation. 
Furthermore, the membrane practical application ability was evaluated by $\mathrm{pH}$ stability, separation of various mixtures, separation at different temperatures/ionic concentrations, and long-term usability. Figure $7 \mathrm{a}$ shows the membrane $\mathrm{CA}$ and underwater oil $\mathrm{CA}$ after immersing in different $\mathrm{pH}$ solutions for $24 \mathrm{~h}$. As illustrated in Figure 7a, the CA increased, and underwater oil CA decreased dramatically with $\mathrm{pH}$ value less than 4.0 , indicating the membrane poor stability properties in strong acid conditions. However, the membranes held the superhydrophilic and underwater superoleophobic properties with $\mathrm{pH}$ values larger than 5.0, suggesting good stability against alkaline conditions. The rejection rate for olive oil, cooking oil, and lubricant oil were all higher than $99.8 \%$ with acceptance flux higher than $2050 \mathrm{Lm}^{-2} \mathrm{~h}^{-1}$ (Figure $7 \mathrm{~b}$ ). As shown in Figure 7c, the mixture temperature affected slightly the separation efficiency due to the change in oil-in-water solubility with temperature increases [45]. Nevertheless, the separation efficiency was still higher than $99.7 \%$, indicating that the membrane could be used in a broad temperature range. In the practical separation process, ions always existed in filtrate. Figure $7 \mathrm{c}$ also shows that $\mathrm{NaCl}$ (model ion) concentrations had no significant influence on the membrane performance, demonstrated by the separation efficiency higher than $99.8 \%$. The long-term usability is a key indicator for practical application. As shown in Figure $7 \mathrm{~d}$, the membrane could keep the flux and separation efficiency for $80 \mathrm{~min}$ without an obvious decrease for the model oil/water mixture (olive oil), suggesting its superior antifouling properties without sacrificing the product quality and productivity. This outcome was contributed to by the water layer formed between the oil and the membrane [46]. Thus, the membrane holds a good anti-fouling property and can be applied for long-term use. Ultimately, the results above suggest that the core-organic/inorganic membrane has great potential in practical oil/water separation.
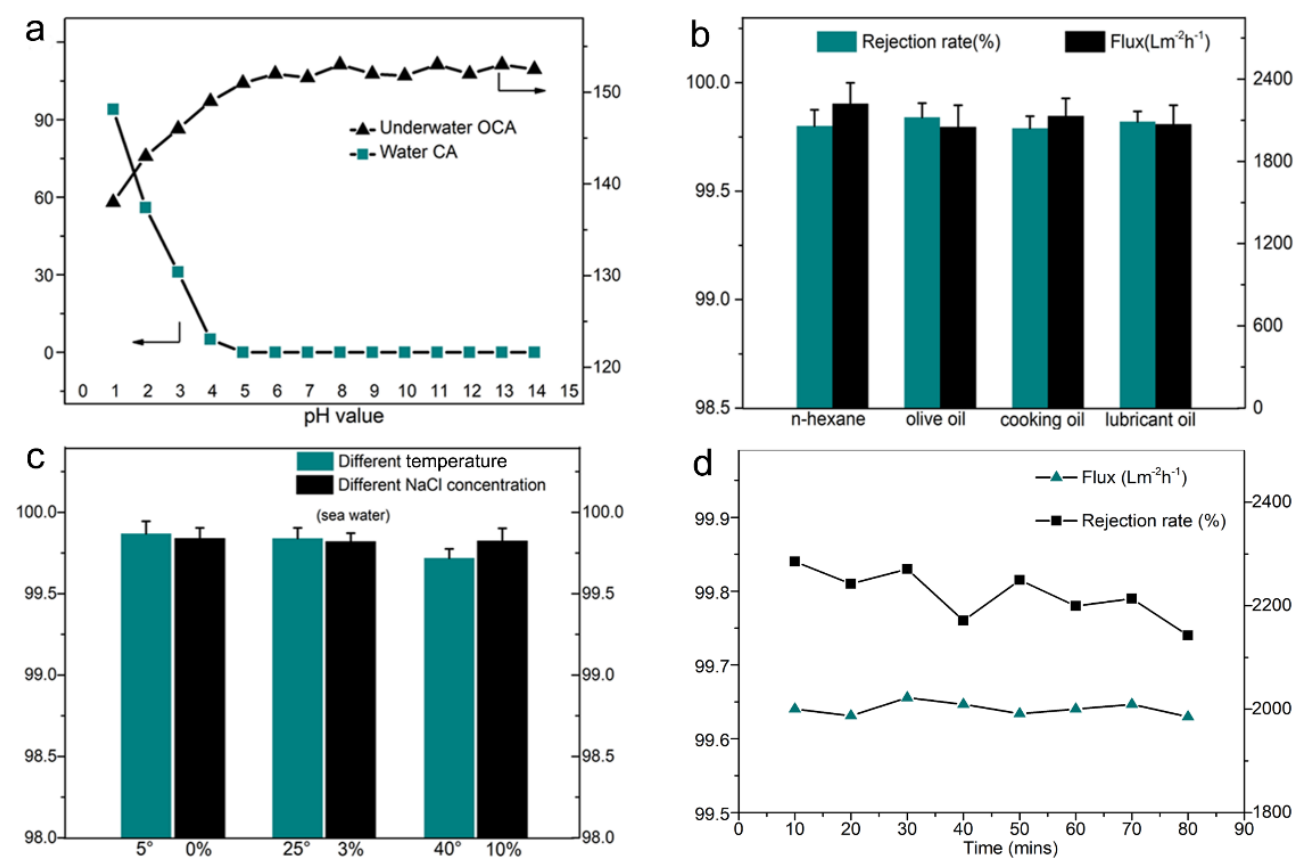

Figure 7. The oil/water mixture separation process: (a) the water CA and underwater oil CA under different $\mathrm{pH}$ value conditions; (b) the rejection rate and flux of different oil/water mixtures; (c) the rejection rate under different penetrating temperature and $\mathrm{NaCl}$ concentration (olive oil as the model oil); (d) the rejection rate and flux under $80 \mathrm{~min}$ (olive oil as the model oil).

\subsection{The Proposed Formation Mechanism of P/CuO Nano-Sheet Membrane}

Based on the morphology and structure characterization, the formation mechanism of core-shell $\mathrm{P} / \mathrm{CuO}$-nanosheet was proposed to give more insightful opinions for future researchers and applications: The polymer and inorganic salt dissolved together to fabricate a PVDF-HFP/Cu( $\left(\mathrm{CH}_{3} \mathrm{COO}\right)_{2}$ composite nanofibrous backbone (Figure 8). After the heating process, the $\mathrm{Cu}\left(\mathrm{CH}_{3} \mathrm{COO}\right)_{2}$ was transferred to 
$\mathrm{CuO}$, which provided the sites where $\mathrm{CuO}$ grew. Then, the resulting $\mathrm{P} / \mathrm{CuO}$ membrane was immersed into the $\mathrm{CuO}$ growth medium. The growth medium was cuprammonia, and a simple preparation process was carried out, as seen in Figure S5. With the temperature increase, the reaction went to the direction of producing $\mathrm{Cu}(\mathrm{OH})_{2}$. Subsequently, the $\mathrm{Cu}\left(\mathrm{NH}_{3}\right)_{4}{ }^{2+}$ dehydrating reaction formed the crystal nucleus of the $\mathrm{CuO}$ nanosheet at the interface between the nanofiber and the growth medium. After the formation of the crystal nucleus, the original granules had a random orientation at the early stage of nucleation growth. After that, the adjacent original granules rotated and shared the same crystallographic orientation driven by the thermal dynamic force, leading to the formation of the $\mathrm{CuO}$ nanosheet [47]. The reactions in the hydrothermal process are speculated as follows:

$$
\begin{aligned}
\left(\mathrm{Cu}\left(\mathrm{NH}_{3}\right)_{4}\right)^{2+}+2 \mathrm{OH}^{-} & \rightarrow \mathrm{Cu}(\mathrm{OH})_{2}+4 \mathrm{NH}_{3} \\
\mathrm{Cu}(\mathrm{OH})_{2} & \rightarrow \mathrm{CuO}
\end{aligned}
$$

or

$$
\begin{aligned}
& \mathrm{NH}_{3}+\mathrm{H}_{2} \mathrm{O} \rightarrow \mathrm{NH}^{4+}+\mathrm{OH}^{-} \\
& 2 \mathrm{OH}^{-}+\mathrm{Cu}^{2+} \rightarrow \mathrm{CuO}+\mathrm{H}_{2} \mathrm{O}
\end{aligned}
$$

or

$$
\begin{aligned}
\mathrm{Cu}^{2+}+\mathrm{OH}^{-} & \rightarrow \mathrm{Cu}(\mathrm{OH})_{2} \\
\mathrm{Cu}(\mathrm{OH})^{2} & \rightarrow \mathrm{CuO}
\end{aligned}
$$

These reactions may happen simultaneously, synergistically forming the $\mathrm{CuO}$ nanosheet on the PVDF-HFP nanofiber backbone.

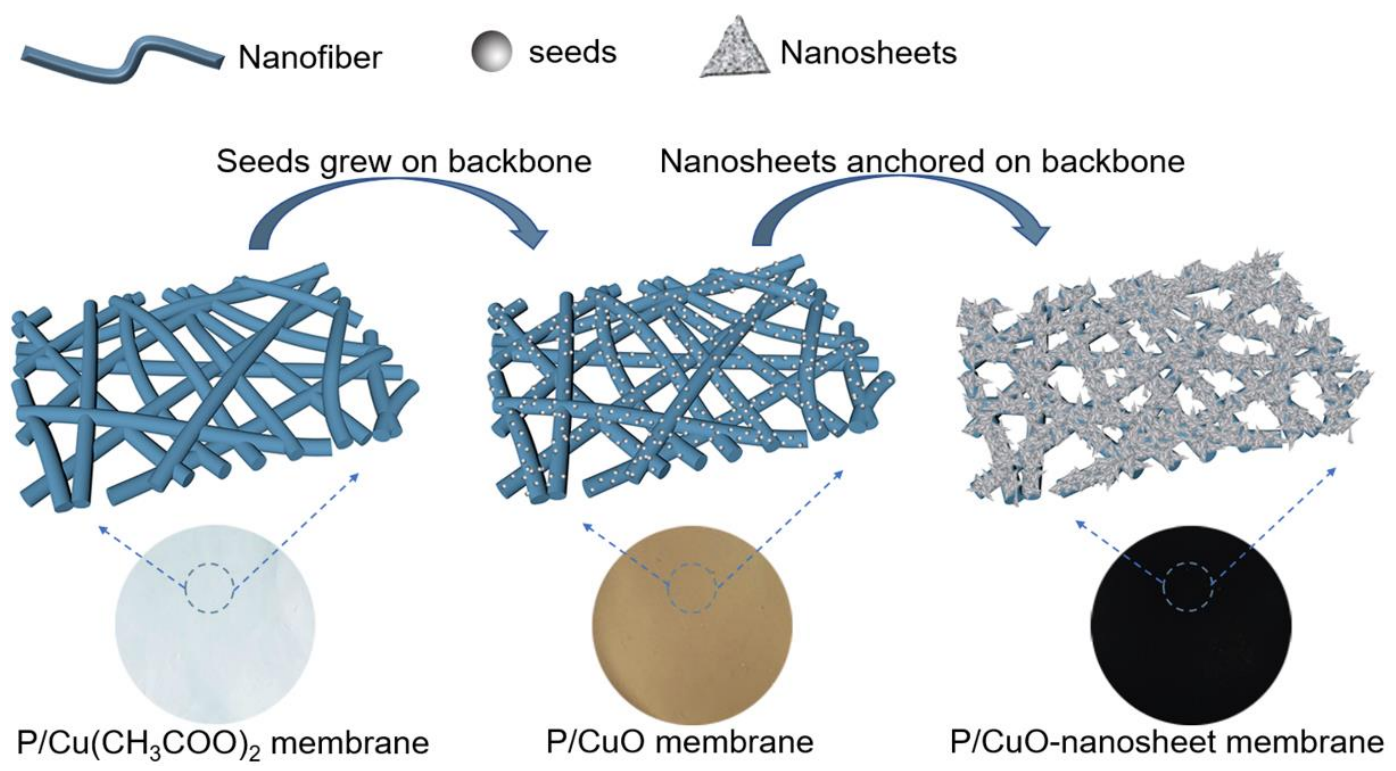

Figure 8. The schematic diagram of proposed formation mechanism for $\mathrm{P} / \mathrm{CuO}$ nanosheet membrane.

\section{Conclusions}

We have successfully demonstrated a core-shell organic/inorganic composite membrane featuring superhydrophilic, underwater superoleophobic, anti-fouling, super-flexible, and alkaline resistance properties. As a result of these synergic integrated advantages, this delicate structure membrane shows excellent oil rejection rates under different filtration temperatures and ionic concentrations. Notably, the easily prepared membrane, using a continuous spinning technology plus low temperature hydrothermal process, exhibits high product quality by rejecting oils with high efficiency, high productivity without obvious fouling tendency at a relatively low pressure driven process even under various 
conditions, and a longer usability period. Therefore, it is of great significance to believe this strategy can become a general and alternative approach to fabricate functional core-shell structure membranes that shows potential applications in practical oil/water separation and other aspects.

Supplementary Materials: The following are available online at http:/www.mdpi.com/2073-4360/11/6/974/s1.

Author Contributions: Conceptualization, Z.L. (Zhi Liu) and H.B.; Methodology, D.D.S.; Investigation, Z.L. (Zhi Liu), D.Q., J.Z., and Z.L. (Zhengtao Li); Writing-Original Draft Preparation, Z.L. (Zhi Liu) and Q.F.; Writing-Review \& Editing, H.B. and D.D.S.; Supervision, Z.L. (Zhi Liu), H.B. and D.D.S.; Project Administration, Z.L. (Zhi Liu); Funding Acquisition, Z.L. (Zhi Liu).

Funding: This research was funded by Anhui Provincial Natural Science Foundation (1908085QE223), the Pre-research Project of the China National Natural Science Foundation of Anhui Polytechnic University (2019yyzr06), and the Anhui Province College Key Laboratory of Textile Fabrics (2018AKLTF07).

Conflicts of Interest: The authors declare no conflict of interest.

\section{References}

1. Judd, S.J. Membrane technology costs and me. Water Res. 2017, 122, 1-9. [CrossRef]

2. Logan, B.E.; Elimelech, M. Membrane-based processes for sustainable power generation using water. Nature 2012, 488, 313. [CrossRef] [PubMed]

3. Shannon, M.A.; Bohn, P.W.; Elimelech, M.; Georgiadis, J.G.; Mariñas, B.J.; Mayes, A.M. Science and technology for water purification in the coming decades. Nature 2008, 452, 301. [CrossRef] [PubMed]

4. Thavasi, V.; Singh, G.; Ramakrishna, S. Electrospun nanofibers in energy and environmental applications. Energy Environ. Sci. 2008, 1, 205-221. [CrossRef]

5. Rogers, J.A.; Lagally, M.G.; Nuzzo, R.G. Synthesis, assembly and applications of semiconductor nanomembranes. Nature 2011, 477, 45. [CrossRef]

6. Gin, D.L.; Noble, R.D. Designing the next generation of chemical separation membranes. Science 2011, 332, 674-676. [CrossRef]

7. Jiang, S.; Chen, Y.; Duan, G.; Mei, C.; Greiner, A.; Agarwal, S. Electrospun nanofiber reinforced composites: A review. Polym. Chem. 2018, 9, 2685-2720. [CrossRef]

8. Liu, Z.; Zhao, J.; Li, W.; Xing, J.; Xu, L.; He, J. Humidity-induced porous poly (lactic acid) membrane with enhanced flux for oil-water separation. Adsorpt. Sci. Technol. 2018. [CrossRef]

9. Bai, H.; Liu, Z.; Sun, D.D. Hierarchically multifunctional $\mathrm{TiO}_{2}$ nano-thorn membrane for water purification. Chem. Commun. 2010, 46, 6542-6544. [CrossRef]

10. Bai, H.; Liu, L.; Liu, Z.; Sun, D.D. Hierarchical 3D dendritic $\mathrm{TiO}_{2}$ nanospheres building with ultralong 1D nanoribbon/wires for high performance concurrent photocatalytic membrane water purification. Water Res. 2013, 47, 4126-4138. [CrossRef]

11. Ma, W.; Zhang, M.; Liu, Z.; Kang, M.; Huang, C.; Fu, G. Fabrication of highly durable and robust superhydrophobic-superoleophilic nanofibrous membranes based on a fluorine-free system for efficient oil/water separation. J. Membr. Sci. 2019, 570, 303-313. [CrossRef]

12. Dzenis, Y. Spinning continuous fibers for nanotechnology. Science 2004, 304, 1917-1919. [CrossRef]

13. Gould, J. Learning from nature's best. Nature 2015, 519, 2-3. [CrossRef]

14. Si, Y.; Fu, Q.; Wang, X.; Zhu, J.; Yu, J.; Sun, G.; Ding, B. Superelastic and superhydrophobic nanofiber-assembled cellular aerogels for effective separation of oil/water emulsions. ACS Nano 2015, 9, 3791-3799. [CrossRef] [PubMed]

15. Feng, L.; Zhang, Z.; Mai, Z.; Ma, Y.; Liu, B.; Jiang, L.; Zhu, D. A super-hydrophobic and super-oleophilic coating mesh film for the separation of oil and water. Angew. Chem. Int. Ed. 2004, 43, 2012-2014. [CrossRef] [PubMed]

16. Li, J.; Kang, R.; Tang, X.; She, H.; Yang, Y.; Zha, F. Superhydrophobic meshes that can repel hot water and strong corrosive liquids used for efficient gravity-driven oil/water separation. Nanoscale 2016, 8, 7638-7645. [CrossRef]

17. Ma, Q.; Cheng, H.; Fane, A.G.; Wang, R.; Zhang, H. Recent development of advanced materials with special wettability for selective oil/water separation. Small 2016, 12, 2186-2202. [CrossRef] [PubMed]

18. Xu, Z.; Miyazaki, K.; Hori, T. Fabrication of polydopamine-coated superhydrophobic fabrics for oil/water separation and self-cleaning. Appl. Surf. Sci. 2016, 370, 243-251. [CrossRef] 
19. Gao, X.; Xu, L.; Xue, Z.; Feng, L.; Peng, J.; Wen, Y.; Wang, S.; Zhang, X. Dual-scaled porous nitrocellulose membranes with underwater superoleophobicity for highly efficient oil/water separation. Adv. Mater. 2014, 26, 1771-1775. [CrossRef] [PubMed]

20. Padaki, M.; Murali, R.S.; Abdullah, M.S.; Misdan, N.; Moslehyani, A.; Kassim, M.A.; Hilal, N.; Ismail, A.F. Membrane technology enhancement in oil-water separation. A review. Desalination 2015, 357, $197-207$. [CrossRef]

21. Jiang, S.; Agarwal, S.; Greiner, A. Low-Density Open Cellular Sponges as Functional Materials. Angew. Chem. Int. Ed. 2017, 56, 15520-15538. [CrossRef] [PubMed]

22. Zhou, S.; Zhou, G.; Jiang, S.; Fan, P.; Hou, H. Flexible and refractory tantalum carbide-carbon electrospun nanofibers with high modulus and electric conductivity. Mater. Lett. 2017, 200, 97-100. [CrossRef]

23. Tao, M.; Xue, L.; Liu, F.; Jiang, L. An intelligent superwetting PVDF membrane showing switchable transport performance for oil/water separation. Adv. Mater. 2014, 26, 2943-2948. [CrossRef]

24. Wong, T.S.; Kang, S.H.; Tang, S.K.Y.; Smythe, E.J.; Hatton, B.D.; Grinthal, A.; Aizenberg, J. Bioinspired self-repairing slippery surfaces with pressure-stable omniphobicity. Nature 2011, 477, 443. [CrossRef] [PubMed]

25. Chu, Z.; Feng, Y.; Seeger, S. Oil/water separation with selective superantiwetting/superwetting surface materials. Angew. Chem. Int. Ed. 2015, 54, 2328-2338. [CrossRef] [PubMed]

26. Zhou, C.; Cheng, J.; Hou, K.; Zhao, A.; Pi, P.; Wen, X.; Xu, S. Superhydrophilic and underwater superoleophobic titania nanowires surface for oil repellency and oil/water separation. Chem. Eng. J. 2016, 301, $249-256$. [CrossRef]

27. Xue, Z.; Wang, S.; Lin, L.; Chen, L.; Liu, M.; Feng, L.; Jiang, L. A novel superhydrophilic and underwater superoleophobic hydrogel-coated mesh for oil/water separation. Adv. Mater. 2011, 23, 4270-4273. [CrossRef]

28. Liu, X.; Zhou, J.; Xue, Z.; Gao, J.; Meng, J.; Wang, S.; Jiang, L. Clam's shell inspired high-energy inorganic coatings with underwater low adhesive superoleophobicity. Adv. Mater. 2012, 24, 3401-3405. [CrossRef]

29. Yuan, T.; Meng, J.; Hao, T.; Wang, Z.; Zhang, Y. A scalable method toward superhydrophilic and underwater superoleophobic PVDF membranes for effective oil/water emulsion separation. ACS Appl. Mater. Interfaces 2015, 7, 14896-14904. [CrossRef]

30. Zhang, L.; Zhong, Y.; Cha, D.; Wang, P. A self-cleaning underwater superoleophobic mesh for oil-water separation. Sci. Rep. 2013, 3, 2326. [CrossRef]

31. Zhang, F.; Zhang, W.B.; Shi, Z.; Wang, D.; Jin, J.; Jiang, L. Nanowire-haired inorganic membranes with superhydrophilicity and underwater ultralow adhesive superoleophobicity for high-efficiency oil/water separation. Adv. Mater. 2013, 25, 4192-4198. [CrossRef] [PubMed]

32. Qin, D.; Liu, Z.; Bai, H.; Sun, D.D.; Song, X. A new nano-engineered hierarchical membrane for concurrent removal of surfactant and oil from oil-in-water nanoemulsion. Sci. Rep. 2016, 6, 24365. [CrossRef] [PubMed]

33. Liu, Z.; Cao, R.; Wei, A.; Zhao, J.; He, J. Superflexible/superhydrophilic PVDF-HFP/CuO-nanosheet nanofibrous membrane for efficient microfiltration. Appl. Nanosci. 2019, 1-10. [CrossRef]

34. Duan, G.; Greiner, A. Air-Blowing-Assisted Coaxial Electrospinning toward High Productivity of Core/Sheath and Hollow Fibers. Macromol. Mater. Eng. 2019, 1800669. [CrossRef]

35. Liu, Z.; Chen, R.; He, J. Active generation of multiple jets for producing nanofibres with high quality and high throughput. Mater. Des. 2016, 94, 496-501. [CrossRef]

36. Liu, Z.; Ang, K.K.J.; He, J. Needle-disk electrospinning inspired by natural point discharge. J. Mater. Sci. 2017, 52, 1823-1830. [CrossRef]

37. Lee, S.S.; Bai, H.; Liu, Z.; Sun, D.D. Optimization and an insightful property-activity study of electrospun $\mathrm{TiO}_{2} / \mathrm{CuO}$ composite nanofibers for efficient photocatalytic $\mathrm{H}_{2}$ generation. Appl. Catal. B Environ. 2013, 140, 68-81. [CrossRef]

38. Wang, T.; Zhai, Y.; Zhu, Y.; Peng, C.; Xu, B.; Wang, T.; Li, C.; Zeng, G. Influence of temperature on nitrogen fate during hydrothermal carbonization of food waste. Bioresour. Technol. 2018, 247, 182-189. [CrossRef]

39. An, H.; Li, Y.; Long, P.; Gao, Y.; Qin, C.; Cao, C.; Feng, Y.; Feng, W. Hydrothermal preparation of fluorinated graphene hydrogel for high-performance supercapacitors. J. Power Sources 2016, 312, 146-155. [CrossRef]

40. Chen, A.; Long, H.; Li, X.; Li, Y.; Yang, G.; Lu, P. Controlled growth and characteristics of single-phase $\mathrm{Cu}_{2} \mathrm{O}$ and $\mathrm{CuO}$ films by pulsed laser deposition. Vacuum 2009, 83, 927-930. [CrossRef]

41. Zhao, Y.; Wang, C.; Wallace, G.G. Tin nanoparticles decorated copper oxide nanowires for selective electrochemical reduction of aqueous $\mathrm{CO}_{2}$ to CO. J. Mater. Chem. A 2016, 4, 10710-10718. [CrossRef] 
42. Zhu, J.; Li, D.; Chen, H.; Yang, X.; Lu, L.; Wang, X. Highly dispersed CuO nanoparticles prepared by a novel quick-precipitation method. Mater. Lett. 2004, 58, 3324-3327. [CrossRef]

43. Meghana, S.; Kabra, P.; Chakraborty, S.; Padmavathy, N. Understanding the pathway of antibacterial activity of copper oxide nanoparticles. RSC Adv. 2015, 5, 12293-12299. [CrossRef]

44. Lee, C.H.; Tiwari, B.; Zhang, D.; Yap, Y.K. Water purification: Oil-water separation by nanotechnology and environmental concerns. Environ. Sci. Nano 2017, 4, 514-525. [CrossRef]

45. Shiu, W.Y.; Bobra, M.; Bobra, A.M.; Maijanen, A.; Suntio, L.; Mackay, D. The water solubility of crude oils and petroleum products. Oil Chem. Pollut. 1990, 7, 57-84. [CrossRef]

46. Wang, B.; Liang, W.; Guo, Z.; Liu, W. Biomimetic super-lyophobic and super-lyophilic materials applied for oil/water separation: A new strategy beyond nature. Chem. Soc. Rev. 2015, 44, 336-361. [CrossRef]

47. Zhang, Z.; Sun, H.; Shao, X.; Li, D.; Yu, H.; Han, M. Three-Dimensionally Oriented Aggregation of a Few Hundred Nanoparticles into Monocrystalline Architectures. Adv. Mater. 2005, 17, 42-47. [CrossRef]

(C) 2019 by the authors. Licensee MDPI, Basel, Switzerland. This article is an open access article distributed under the terms and conditions of the Creative Commons Attribution (CC BY) license (http://creativecommons.org/licenses/by/4.0/). 